\title{
Supplemental Information to Protein Conformation-Induced Modulation of Ligand Binding Kinetics: A Femtosecond Mid-IR Study of Nitric Oxide Binding Trajectories in Myoglobin
}

\author{
Seongheun Kim and Manho Lim* \\ Department of Chemistry, Pusan National University, Busan 609-735 Korea
}

Received January 13, 2005; Email: mhlim@pusan.ac.kr

\section{Experimental details}

The details of the time-resolved mid-IR spectrometer used here are described elsewhere. ${ }^{1}$ Briefly, two identical home-built optical parametric amplifiers (OPA), pumped by a commercial Ti:sapphire amplifier, are used to generate a visible pump pulse and a mid-IR probe pulse. The signal pulse of one OPA is frequency doubled in a type-I, 1-mm-thick BBO crystal to generate a tunable pump pulse in the visible region. The signal and idler pulse of the other OPA are difference frequency mixed in a 1.5 -mm-thick, type-I $\mathrm{AgGaS}_{2}$ crystal to yield a tunable midIR pulse $\left(110 \mathrm{fs}, 160 \mathrm{~cm}^{-1}, 1 \mu \mathrm{J}\right)$. A small portion of this pulse is used to probe transient mid-IR absorbance of the sample photolyzed by $580 \mathrm{~nm}$ pulses. The polarization of the pump pulse was set at the magic angle $(54.7$ relative to the probe pulse to recover the isotropic absorption spectrum. The broadband transmitted probe pulse is detected with a 64-elements $\mathrm{N}_{2}(l)$ cooled $\mathrm{HgCdTe}$ array detector. The array detector is mounted in the focal plane of a $320 \mathrm{~mm}$ monochromator with a $150 \mathrm{l} / \mathrm{mm}$ grating, resulting in a spectral resolution of ca. $1.2 \mathrm{~cm}^{-1} / \mathrm{pixel}$ at $1800 \mathrm{~cm}^{-1}$. The signals from each of the detector elements were amplified with a home-built 64-channel amplifier and digitized by a 12-bit ADC. Due to the excellent short-term stability of the IR light source $(<0.5 \% \mathrm{rms})$, the pump-induced change in the absorbance of the sample $\Delta A$ is routinely computed to a precision $<1 \times 10^{-4} \mathrm{rms}$ after $0.5 \mathrm{sec}$ of signal averaging without single shot referencing with an independent detector. The instrument response function is typically $180 \mathrm{fs}$.

A $12 \mathrm{mM}$ MbNO sample was prepared by dissolving lyophilized skeletal horse myoglobin (Sigma) in deoxygenated $\mathrm{D}_{2} \mathrm{O}$ buffered with $0.1 \mathrm{M}$ potassium phosphate $(\mathrm{pD} 7.4)$. It was reduced with a 2-fold excess of freshly prepared sodium dithionate (Aldrich), and adding an equivalent quantity of $0.1 \mathrm{M}$ degassed sodium nitrite (Aldrich) solution in the presence of an equimolar quantity of $0.1 \mathrm{M}$ sodium dithionate. ${ }^{2}$ All sample preparations were carried out in an ice bath to minimize thermal denaturation. To remove light scattering sources such as dust particles and denatured protein aggregates, samples were filtered through a $0.45-\mu \mathrm{m}$ membrane filter before loading in a gas-tight flowing sample cell with 2-mm-thick $\mathrm{CaF}_{2}$ windows (pathlength; $100 \mu \mathrm{m})$. Throughout the experiments the integrity and concentration of sample was routinely checked using UV-Vis and FT-IR spectroscopy. During data collection the sample cell was flowed sufficiently fast so that each photolyzing laser pulse illuminated a fresh volume of the sample. The sample was prepared in $\mathrm{D}_{2} \mathrm{O}$ to isotopically shift the spectral region of interest to a region with greater IR transmission. The temperature of the sample cell was kept at $283 \leq 1 \mathrm{~K}$.

\section{Band assignment}

The spectral region probed is free from any protein absorption but has a small absorption due to the weak and broad combination of bending and liberation mode of $\mathrm{D}_{2} \mathrm{O}$ at $1555 \mathrm{~cm}^{-1}$ with $\sim 200$ $\mathrm{cm}^{-1}$ full width at half maximum (FWHM) $\left(\varepsilon_{\max }=1.91 \mathrm{M}^{-1} \mathrm{~cm}^{-1}\right){ }^{3}$ Featureless baseline shift, attributed to absorbance change of solvent by thermal relaxation of the photoexcited protein, was modeled by a quadratic polynomial and subtracted for clarity. The peak at the high energy side is blue-shifted while that in the middle is red-shifted from the gas state value, $1876.09 \mathrm{~cm}^{-1}$. The blue-shifted peak is described by a single band and denoted $B_{0}{ }^{5}$ The red-shifted middle peak initially starts from a single feature and evolves toward two bands, and denoted $B_{1}$ and $B_{2}$ (see Figure 1). The peak near $1833 \mathrm{~cm}^{-1}$ is attributed to the $\mathrm{v}=1 \varnothing \mathrm{v}=2$ transition of NO (hot band) because it is replicas of other bands at the high energy side and red-shifted by $30 \leq 2 \mathrm{~cm}^{-1}$, similar to anharmonicity of NO in the gas phase, $27.94 \mathrm{~cm}^{-1}$. As shown in the bottom of Figure 1, the middle peak $\left(B_{1}\right.$ and $\left.B_{2}\right)$ is similar to two photoproduct states observed at $7 \mathrm{~K}^{6}{ }^{6}$ corroborating above assignment. In $\mathrm{MbCO}$ photolysis at cryogenic temperature, significant amount of $B_{0}$ was observed when short illumination of photolysis light was used. ${ }^{7}$ Sample-to-sample variant $B_{0}$ was reported with other photolysis conditions such as extended illumination or using continuous tungsten light source. ${ }^{5,7}$ These indicate that $B_{0}$ is unstable and quickly rebinds or interconverts into other states.

\section{Data analysis}

The spectra were modeled with a sum of tree Gaussians plus their red-shifted replicas. Initially spectral parameters of three bands were allowed to vary independently. Both the center frequency and FWHM changed exponentially in time. Furthermore for a given band, the exponential time constant of the center frequency was similar to that of FWHM. Thus a shared exponential time constant was used for the final fitting of time dependencies of the center frequency and FWHM of each band. These constraints did not affect much the kinetics of the integrated absorbance of each band. In contrast to $B_{0}$ and $B_{1}$, the growth time of $B_{2}$ is much slower than that of total $B$ state (see Figure S1), suggesting that the increase of $B_{2}$ have contribution other than the structural rearrangement such as NO population change due to transitions between $B$ states. Fractional population (Figure 2A) clearly shows transition between $B_{0}$ and $B_{1}$, and trend that $B_{1}$ and $B_{2}$ approach equilibrium each other.

About $40 \%$ of the main band appears in the hot band (see Figure 1), which is higher than that observed in the photolysis of $\mathrm{MbCO}$ where $10-16 \%$ was observed in the hot band. ${ }^{8}$ Evidently energy transfer from an impulsive half collision into the NO vibration is more efficient in the photolysis of MbNO than that of MbCO. 9 The relative hot band population can be obtained by 
accounting for both the absorbance cross-sections of fundamental and hot band. Using harmonic oscillator approximation for the absorbance cross-section, ${ }^{10}$ the integrated absorbance for transition from the first excited state, $A_{e}$ and the ground state, $A_{g}$ can be expressed as $A_{e}=2 \varepsilon n_{e}$ and $A_{g}=\varepsilon\left(n_{g}-n_{e}\right)$. Here, $n_{e}$ and $n_{g}$ are the population of the first excited state and the ground state, respectively and $\varepsilon$ is the absorbance cross-section for the ground state transition. The nascent vibrationally excited NO population $(15 \pm 4 \%)$ and the vibrational relaxation time of NO ( $200 \mathrm{ps})$ were obtained from the kinetics of the relative amplitude of the red-shifted absorbance. These are independent of the kinetic model used. For all the kinetic model describing the kinetic behavior of the $B$-bands, the rates for the transitions and rebinding of NO are assumed to be independent of its vibrational energy. ${ }^{11}$ The time dependent rebinding rate, $k_{g r}(t)$ is given by introducing a time dependent barrier $E(t)$ into the Arrhenius equation, $k(t)=A$ $\exp [-E(t) / R T]$, with $E(t)=\left(E_{0}-E_{e q}\right) \exp \left(-k_{b a r} t\right)+E_{\text {eq }}$, where $k_{\text {bar }}$ is the rate constant for the variation in the barrier height from its initial height $E_{0}$ to its equilibrium value $E_{e q}{ }^{1,12}$

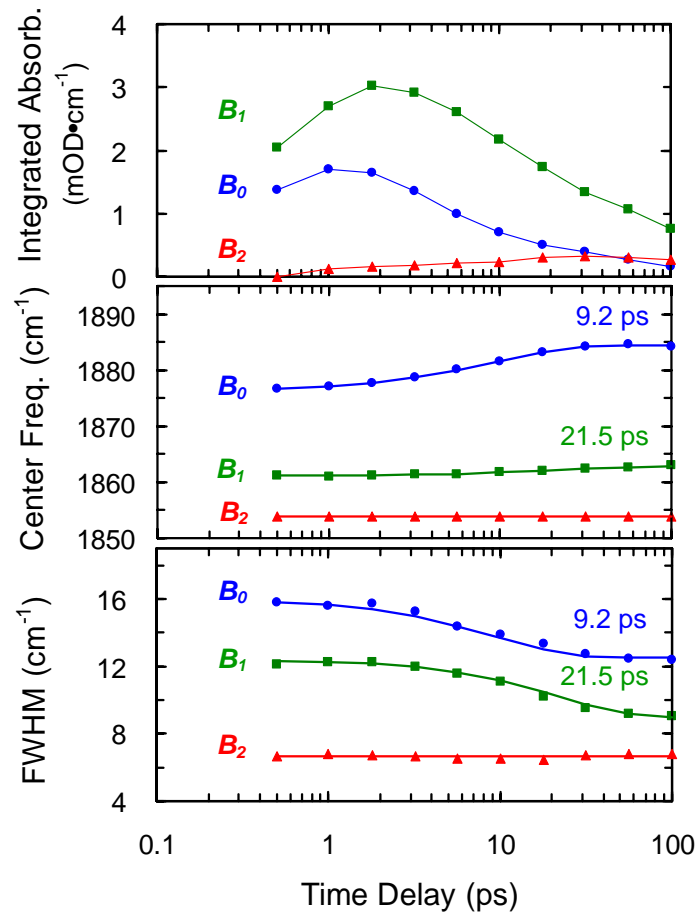

Figure S1. The fitted parameters for three Gaussians. The thick solid lines in the middle and bottom panels are single exponential fits to the fitted spectral parameters (symbols). The thin solid lines in the top panel are to guide eyes.

Since bound NO is severely bent, ${ }^{13}$ the translational energy of departing NO after photoexcitation is likely smaller than that of $\mathrm{CO}$, thus, as observed, most of nascent photolyzed $\mathrm{NO}$ goes to nearby bound site $\left(B_{0}\right.$ and $\left.B_{1}\right)$ rather than farther $B_{2}$. As suggested, $B_{1}$ and $B_{2}$ likely correspond to photolyzed NO in adjacent sites in the heme pocket found in MD simulations with almost opposite orientation., 14 Time-resolved polarization anisotropy experiment would be useful to test above suggestions by probing the orientational dynamics of photolyzed NO. MD simulations extracting the rebinding trajectories of photolyzed $\mathrm{NO}$ can add further insight on the rebinding of the ligand to $\mathrm{Mb}$.

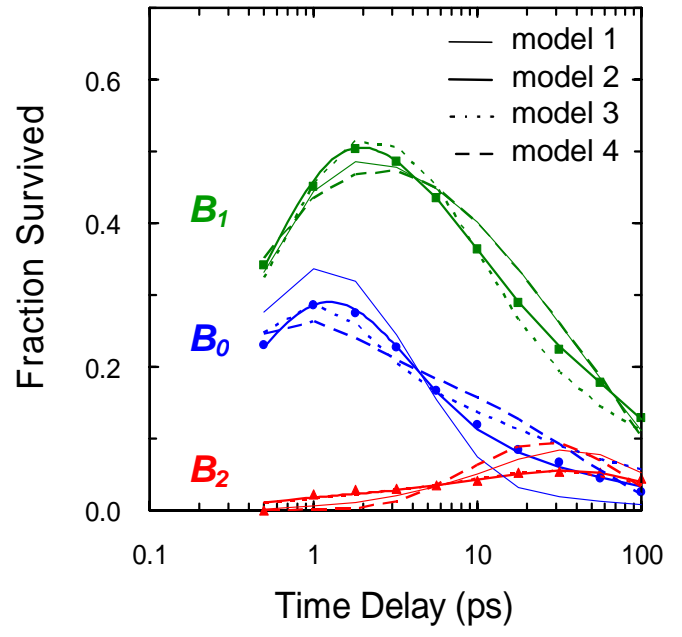

Figure S2. Time dependence of the integrated $B$ state absorbance. The solid lines represent the fit to the integrated absorbances using various models described in the text with an initial rise time.

In the kinetic model 1 , all three $B$ states can directly rebind and interconvert each other. It is the most general kinetic model with time-independent rates. Because the time-independent rates for all the possible interconversion and rebinding cannot reproduce the time-dependence of the integrated $B$ state absorbance, we began to incorporate time dependence in the rates. Recent experiment on photolyzed $\mathrm{CO}$ from $\mathrm{HbCO}$ and $\mathrm{MbCO}$, where ligands interconvert without rebinding, showed that the interconversion is well described by time-independent rates. ${ }^{15}$ Moreover the dynamics of geminate rebinding of photolyzed NO from $\mathrm{MbNO}$, probed by the recovery kinetics of $\mathrm{MbNO}$, has been described by time-dependent rebinding rate. ${ }^{1,12}$ Therefore, it is reasonable to incorporate time-dependence only in the rebinding rate and assume interconversion rates time-independent. In our kinetic model, only the rebinding rate is allowed to become time dependent. The kinetic model 2 (model 3$)$ incorporates the timedependent rate into the rebinding from $B_{1}\left(B_{0}\right)$. As shown in Figures $2 \mathrm{~A}$ and $\mathrm{S} 2$, only model 2 well reproduced time dependence of the integrated $B$-state absorbance. Since the nascent population of $B_{2}$ is negligible, kinetic model with timedependent rebinding from $B_{2}$ is not discussed. However, a model with time-dependent rebinding from $B_{2}$ results in poor fit and unphysical parameters. For the sake of completeness, models with time-dependent interconversion rates and time-independent rebinding rate has been used to fit the data. A kinetic model 4 incorporates the time-dependent rates into the interconversion rates between $B$-states, $B_{1}$ and $B_{2}$ as well as $B_{1}$ and $B_{0}$. As can be seen in figure $\mathrm{S} 2$, the fit with model 4 severely deviates from the data, which suggests that time-dependent rebinding is necessary to reproduce the data.

Whereas model 2 reproduces the data very well, a more sophisticated kinetic scheme may need to be tested. For this we have parameterized kinetics of each $B$-state using a biexponential function and the fitted parameters are given in Table S1. With these parameters, the kinetic behavior of our data can be obtained and any kinetic schemes can be readily tested. 
Table S1. The fitted parameters of the kinetics for the each $B$-state using a biexponential function, $a_{1} \exp \left(-\mathrm{t} / \tau_{1}\right)+a_{2} \exp \left(-\mathrm{t} / \tau_{2}\right)$, plus an initial rise time, $0.52 \mathrm{ps}$

\begin{tabular}{|l|l|l|l|l|}
\hline & $a_{1}$ & $\tau_{1}(\mathrm{ps})$ & $a_{2}$ & $\tau_{2}(\mathrm{ps})$ \\
\hline$B_{0}$ & 0.29 & 3.3 & 0.12 & 51 \\
\hline$B_{1}$ & 0.36 & 13.8 & 0.20 & 271 \\
\hline$B_{2}$ & -0.05 & 10.6 & 0.06 & 257 \\
\hline
\end{tabular}

Acknowledgement. We wish to thank Dr Philip A. Anfinrud for very valuable discussions and suggestions. We also thank Geunyeong Jin and Jeonghee Heo for their help with sample preparation. This work was supported by grants from the KOSEF through CIMS (POSTECH).

\section{References}

(1) Kim, S.; Jin, G.; Lim, M., J. Phys. Chem. B 2004, 108, 20366-20375.

(2) Moller, J. K. S.; Skibsted, L. H., Chem. Rev. 2002, 102, 1167-1178,

(3) Venyaminov, S. Y.; Prendergast, F. G., Anal. Biochem. 1997, 248, 234-245.

(4) Herzberg, G., Spectra of diatomic molecules. Van Nostrand Reinhold Ltd.: New York, 1950

(5) Alben, J. O.; Beece, D.; Bowne, S. F.; Doster, W.; Eisenstein, L.;

Frauenfelder, H.; Good, D.; McDonald, J. D.; Marden, M. C.; Mo, P. P.; Reinisch, L.; Reynolds, A. H.; Shyamsunder, E.; Yue, K. T., Proc. Natl. Acad. Sci. U.S.A. 1982, 79, 3744-3748.

(6) Miller, L. M.; Pedraza, A. J.; Chance, M. R., Biochemistry 1997, 36, 12199 12207.

(7) Abadan, Y.; Chien, E. Y. T.; Chu, K.; Eng, C. D.; Nienhaus, G. U.; Sligar, S. G., Biophys. J. 1995, 68, 2497-2504.

(8) (a) Lim, M.; Jackson, T. A.; Anfinrud, P. A., J. Chem. Phys. 1995, 102, 4355-4366 (b) Sagnella, D. E. Straub, J. E.; Jackson, T. A.; Lim, M. Anfinrud, P. A Proc. Natl. Acad. Sci. U.S. A 1999, 96, 14324-14329.

(9) Anfinrud, P. A.; Han, C.; Hochstrasser, R. M., Proc. Natl. Acad. Sci. U.S.A. 1989, 86, 8387-8391.

(10) Wilson, E. B.; Decius, J. C.; Cross, P. C., Molecular Vibrations: The Theory of Infraed and Raman Vibrational Spectra. Dover: New York, 1955

(11) Meuwly, M.; Becker, O. M.; Stote, R.; Karplus, M., Biophys. Chem. 2002 $98,183-207$

(12) Petrich, J. W.; Lambry, J. C.; Kuczera, K.; Karplus, M.; Poyart, C.; Martin, J. L., Biochemistry 1991, 30, 3975-3987.

(13) Brucker, E. A.; Olson, J. S.; Ikeda-Saito, M.; Phillips, G. N., Jr., Proteins: Struct., Funct., Genet. 1998, 30, 352-356.

(14) Lim, M.; Jackson, T. A.; Anfinrud, P. A., J. Am. Chem. Soc. 2004, 126 7946-7957.

(15) Kim, S.; Lim, M., J. Am. Chem. Soc. 2005, 127, 5786-5787. 
